\title{
Characterization of patients with metastatic non-small-cell lung cancer obtaining long-term benefit from immunotherapy
}

\author{
Giulia Galli ${ }^{\ddagger 1}$, Claudia Proto ${ }^{\ddagger 1}$, Diego Signorelli ${ }^{1}$, Martina Imbimbo ${ }^{1}$, Roberto Ferrara ${ }^{1}$, \\ Arsela Prelaj ${ }^{1}$, Alessandro De Toma1', Monica Ganzinelli ${ }^{1}$, Nicoletta Zilembo ${ }^{1}$, Filippo de \\ Braud $^{1,2}$, Marina C Garassino ${ }^{1}$ \& Giuseppe Lo Russo*,1 (iD \\ ${ }^{1}$ Department of Medical Oncology, Unit of Thoracic Oncology, Fondazione IRCCS Istituto Nazionale dei Tumori, via G Venezian 1 , \\ 20133 Milan, Italy \\ ${ }^{2}$ Department of Medical Oncology \& Hematology, University of Milan, via Festa del Perdono 7, 20122 Milan, Italy \\ *Author for correspondence: Tel.: +39022 390 3829; Fax: +39 022390 2775; giuseppe.lorusso@istitutotumori.mi.it \\ $\ddagger$ Authors contributed equally
}

\begin{abstract}
Aim: A minority of patients gains advantage from immunotherapy (IO). Predictive variables of long-term benefit (LTB) are incompletely understood. Materials \& methods: We retrospectively collected data about metastatic non-small-cell lung cancer patients treated with IO from April 2013 to July 2017. We defined LTB to IO as complete response (CR), partial response (PR) or disease stability as best response and maintaining it for $\geq 12$ months. Results: Thirty-five of the 147 patients had LTB. More LTB patients than controls showed $C R / P R$ as first and best response to IO. Only CR/PR as best response to IO retained association to LTB at multivariate analyses. Conclusion: Objective response appears as a central factor for LTB from IO.
\end{abstract}

First draft submitted: 5 February 2019; Accepted for publication: 20 June 2019; Published online: 24 July 2019

Keywords: disease response $\bullet$ immunotherapy $\bullet$ long-term benefit $\bullet$ non-small-cell lung cancer $\bullet$ survival

Non-small-cell lung cancer (NSCLC) is the malignancy with the highest mortality worldwide [1].

In recent years, the advent of immunotherapy (IO) has led to a progressive survival prolongation, with positive results in comparison to chemotherapy in all treatment lines [2-8]. One of the most interesting aspects of IO is the possibility of inducing sustained responses in a minority of patients, differently from chemotherapy. Such cases seem to entail a particularly favorable prognosis, and exceptional reports of apparent 'cure' of metastatic disease have been documented. Nonetheless, only a minority of patients derives a benefit from treatment, and cases of long-term survival are even less common [9-11]. Many studies have focused on identifying predictors of response to immune checkpoint inhibitors (ICIs), most of them proving a role for PD-L1 expression and tumor mutational burden [12,13]. In contrast with the considerable amount of evidence about response to IO, data are still lacking about variables potentially associated to long-term benefit (LTB) from treatment, and this small subgroup of patients is still poorly characterized [14].

We aimed at investigating this topic in a retrospective cohort of metastatic NSCLC patients treated with IO at our Institution. With this purpose we divided the population between patients with LTB, patients with shortterm benefit (STB) and progressors (P), in order to compare the distribution of main demographic, clinical and pathological characteristics between such subgroups.

\section{Materials \& methods}

We retrospectively collected data about all consecutive patients with metastatic NSCLC beginning IO at Istituto Nazionale dei Tumori, Milan, Italy, between April 2013 and July 2017. Patients beginning treatment after July 2017 were excluded from the analysis, because their follow-up was considered insufficient to evaluate the duration of response. The cut-off for minimum follow-up length was empirically set at 12 months, as it was expected to be sufficient to observe $\geq 50 \%$ of progressions according to regulatory trials in first and more advanced lines of therapy.

Future Medicine 
Demographic, clinical and pathological data were systematically extracted from institutional database. Data were collected about sex, age, smoking status, disease stage at diagnosis, tumor histology, PD-L1 status (when available), specific ICI prescribed, line of IO, performance status (PS) at the beginning of IO, sites of distant disease at the beginning of IO (as both number of metastatic sites and presence of liver metastases), first and best response to IO, response to previous or subsequent chemotherapy, toxicity of at least moderate grade, neutrophil/lymphocyte ratio (NLR) at the beginning of $\mathrm{IO}$, use of steroids at a dose $\geq 10 \mathrm{mg}$ every day of prednisone or equal within the month after the first administration of $\mathrm{IO}$, use of antibiotics between 1 month before and 3 months after the first administration of IO, radiotherapy in the month before or during IO, and use of metformin, statins and/or acetilsalicilic acid during IO.

Tumor histology was defined by institutional pathologists, according to international guidelines. PD-L1 status was defined as positive, when either having an expression $>1 \%$ at institutional test (DAKO 22C3), or being defined as positive/highly expressed at central testing for patients enrolled in clinical trials (irrespective of the specific test used). NLR was accounted as a categorical variable, with a cut-off of 5 .

All ICIs were included in the analysis. Cases were considered eligible provided that they performed at least one radiologic disease evaluation, or that they undergo unequivocal clinical disease progression after at least two subsequent administrations of IO. Cases receiving only the first dose of ICI and then discontinuing it for worsening of general conditions or death were excluded from the analysis. Patients receiving at least two doses of ICIs were included in the analysis and accounted as $\mathrm{P}$ when discontinuation was due to unequivocal clinical progression. Patients showing a disease progression (PD) as first response and subsequently developing either a disease stability (SD) or a partial response (PR) or a complete response (CR) as best response were classified as pseudo-Ps, irrespective of duration of response.

Disease response was evaluated by Institutional radiologists according to Response Evaluation Criteria for Solid Tumors (RECIST) version 1.1. Toxicity was graded according to Common Terminology Criteria for Adverse Events (CTCAE) version 4.0, and adverse events were considered only if graded $\geq 2$.

Cases were defined to have an LTB from IO if they obtained a CR or a PR or an SD as best response, and maintained it for at least 12 months. Patients were defined to have an STB if they obtained either a CR or a PR or an $\mathrm{SD}$ as best response to IO, but then undergoing PD within the subsequent 12 months. Cases who obtained a progression as best response were defined as $\mathrm{P}$.

After the first analysis comparing LTB patients with controls $(S T B+P)$, in order to exclude patients never responding to IO, we performed a second analysis comparing patients with LTB and STB, thus excluding P. Indeed, in this second analysis the difference between the two subgroups (LTB and STB) consisted only in the duration of benefit from IO.

At the end, we repeated the same analysis comparing the two extreme subgroups of the cohort (LTB and P), as a confirmation of previous findings.

All the patients signed a written informed consent, agreeing to the treatment of personal data for research purposes at some time of their disease history. The study was conducted in accord to the Declaration of Helsinki about ethical principles for medical research involving human subjects.

\section{Statistical methods}

Descriptive statistics were used for the analyzed variables. Differences in their distribution were evaluated through Fisher's exact test or $\chi^{2}$ test, as appropriate. Multivariate analyses were performed with logistic regression, which was applied to variables with $<15 \%$ missing data and univariable $\mathrm{p}<0.1$ Wilcoxon matched pairs test was used to estimate difference between means.

Progression-free survival (PFS) was defined as the time interval between the beginning of IO and PD or death for any cause. Overall survival (OS) was defined as the time interval between the beginning of IO and death for any cause. Patients were right censored at the time of death or last follow-up.

Survival was estimated with Kaplan-Meier method. Duration of follow-up was calculated with reverse KaplanMeier method. Cox regression model was used to compare survival curves. All analyses were two-sided and statistical significance threshold was set at 0.05 .

Statistical analyses were performed with SAS (version 9.4, SAS Institute, NC, USA). 


\section{Results}

\section{Patients characteristics \& concomitant medications}

One hundred and forty-seven patients were identified. Eighty-six of them were males. Median age was 66.6 (range: 30.6-84.5). Most patients (81.0\%) were former or current smokers. PS at the beginning of IO was 0 in $46.9 \%$ of cases, one in $44.2 \%$ of cases, two in $8.9 \%$ of cases. As regards concomitant medications during IO, 19 patients were treated with one or more antibiotic cycles between 1 month before and 3 months after the beginning of IO. Steroid prescription within 1 month after the first administration of ICI was registered in $17.0 \%$ of cases. Thirty-seven patients were treated with acetilsalicilic acid, 21 with a statin, 13 with metformin at any time of IO course.

\section{Tumor characteristics}

NSCLC had a nonsquamous histology (including mixed, not otherwise specified and undifferentiated forms) in 103 cases. Disease stage at diagnosis according to American Joint Committee on Cancer Staging version VII was I in eight cases, II in 13 cases, III in 39 cases and IV in 87 cases. PD-L1 expression on tumor specimen was classified as positive in 46 cases, negative in 34 cases and unknown in 67 cases. The large majority of patients (130/147) had more than one site of distant disease at first administration of ICI; liver metastases were present in 31 cases. A high NLR at the beginning of $\mathrm{IO}$ was registered in $32.0 \%$ of patients.

\section{Treatment characteristics}

IO consisted in an anti-PD-1 agent in 87 cases, in an anti-PD-L1 agent in 52 cases, in a combination anti-PDL1/PD-1 + anti-CTLA4 in seven cases and in an anti-CTLA4 in one case. ICIs were administered in first-line setting in 19 patients, in second-line setting in 63 patients, as a third or more advanced line of therapy in 64 patients. Forty-eight patients developed any grade $\geq 2$ toxicity attributable to IO. Radiation therapy within 1 month before the first administration of ICIs or during IO was performed in $19.7 \%$ of cases.

One hundred and twenty-six patients received at least one previous line of chemotherapy, consisting in a platinum-based doublet in 71 cases, in a taxane in 29 cases, in a single different cytotoxic agent in 25 patients and in a nonplatinum-based doublet in two cases. Best response to chemotherapy was PR in 36 patients, SD in 28 patients and PD in the remaining ones. Median PFS was 2.9 months.

A subsequent line of chemotherapy after the conclusion of IO was administered in 33 cases. Seventeen patients received a taxane as single agent or in combination with either a cytotoxic or a biologic drug; eight patients received a platinum-based doublet; and the remaining patients received gemcitabine or navelbine. Median PFS was 2.5 months.

In the global population, first response to IO consisted in a PR in 22 cases (15.0\%), in an SD in 44 cases (29.9\%) and in a PD in the remaining ones (55.1\%). Among the patients obtaining PR, two CRs were subsequently documented as best response to IO; both these patients were among those obtaining LTB from IO. Fourteen cases of pseudoprogression were identified; best response following initial PD was PR in four patients, SD in the remaining ones. Two pseudo-Ps developed an LTB from IO, and best response was PR in both these cases.

Apart from the two CRs, best response to IO was PR in 31 patients, SD in 47 and PD in the remaining ones. Median time to best response was 1.8 months (range: 0.2-17.5 months).

Most patients (106/147) discontinued IO due to PD; a limited number of discontinuations for toxicity (9/147) and for regular conclusion of treatment plan (10/147) were reported. IO was ongoing at the time of database lock in 22 cases.

Median PFS was 2.9 months (95\% CI: 2.3-3.8 months); and median OS was 11.2 months (95\% CI: 8.113.9 months).

\section{Comparison between LTB patients \& controls in the global population}

After a median follow-up of 28.5 months, 35 patients obtaining LTB from IO were identified. As expected according to the definition of LTB, LTB patients showed a significantly longer median PFS (21.0 vs 2.0 months, $\mathrm{p}<0.0001)$ and OS (not reached vs 7.0 months, $\mathrm{p}<0.0001)$ than controls $(\mathrm{STB}+\mathrm{P})$ (Figure 1).

Median PFS of previous and subsequent lines of therapy were not significantly different between the subgroups of patients with or without LTB ( $\mathrm{p}=0.6376$ and 1.0000 , respectively).

None of the analyzed demographic, clinical and pathological variables showed a different distribution between the two subgroups of patients, except for the NLR (odds radio [OR]: 0.3583; 95\% CI: 0.1372-0.9354; $\mathrm{p}=0.0378$ ), the use of steroids at the beginning of IO (OR: 0.05364; 95\% CI: 0.00317-0.9078; $\mathrm{p}=0.0023$ ) and the evidence 

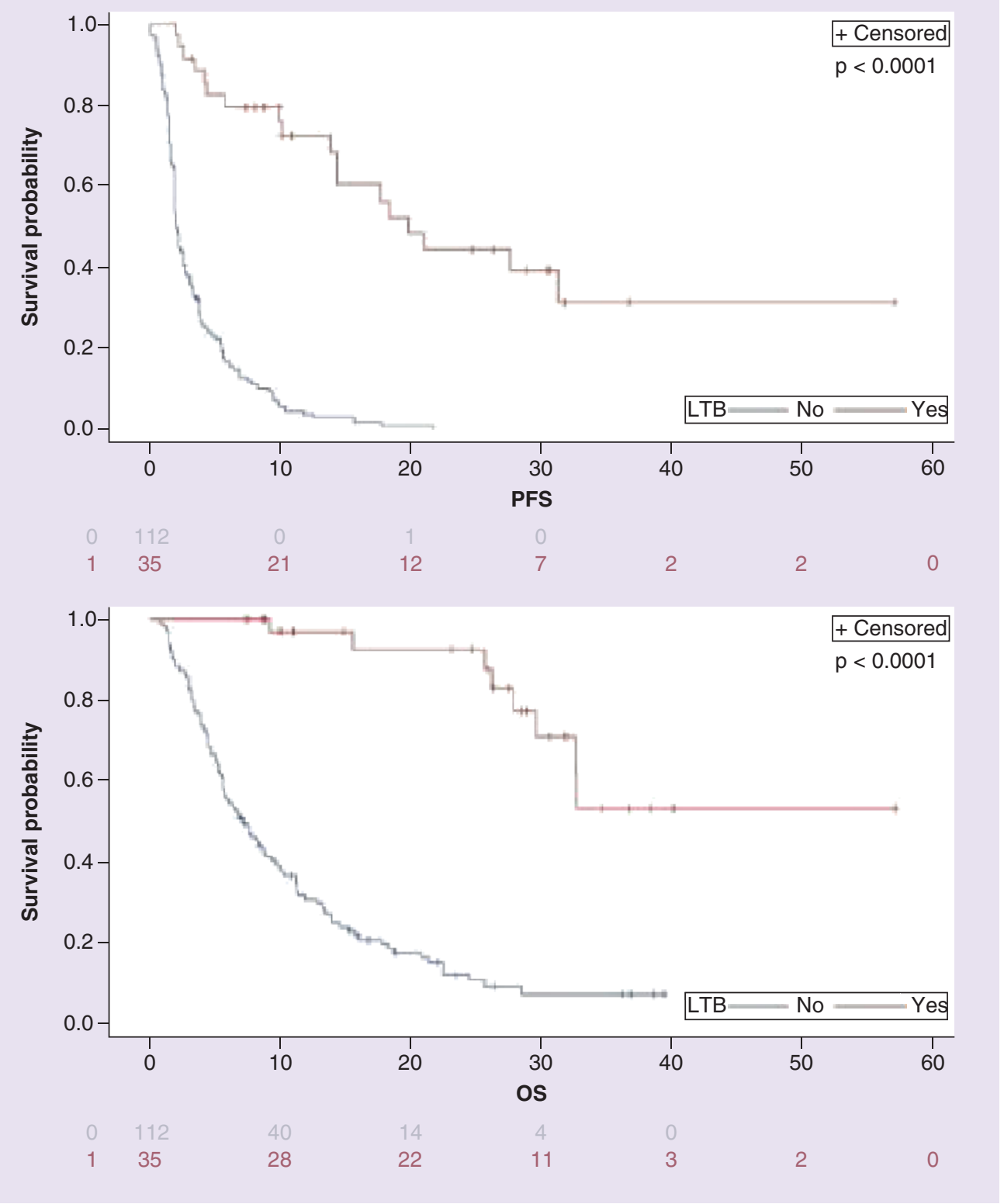

Figure 1. Kaplan-Meier curves of progression-free and overall survival according to long-term benefit in the global population.

LTB: Long-term benefit; OS: Overall survival; PFS: Progression-free survival.

of CR or PR as first (OR of SD or PD vs CR or PR: 5.322; 95\% CI: 2.051-13.810; $\mathrm{p}=0.0007$ ) and best response to IO (OR for SD or PD vs CR or PR: 8.313, 95\% CI: 3.484-19.830; p < 0.0001). Notably, as regards the result about steroid administration, none of the patients in the LTB group received steroids at beginning of IO. At multivariate analysis, only the evidence of $\mathrm{CR}$ or $\mathrm{PR}$ as best response retained a significantly different distribution between subgroups (OR for SD or PD vs CR or PR: 0.124; 95\% CI: 0.034-0.453; p = 0.0016). Detailed results are reported in Tables 1 and 2.

In order to exclude that potential associations between clinical variables and LTB could be masked by the effect of response, we repeated the multivariate analysis excluding response-related variables. These results confirmed the absence of significant associations for NLR ( $\mathrm{p}=0.1828)$, for the use of steroids $(\mathrm{p}=0.1418)$ and for PS at baseline $(\mathrm{p}=0.4050$; data not shown in Tables). 
Table 1. Univariate analyses comparing cases with long-term benefit and controls in the global population.

\begin{tabular}{|c|c|c|c|}
\hline Characteristics & $\begin{array}{l}\text { LTB, } N(\%) \\
N=35\end{array}$ & $\begin{array}{l}\text { STB + P, N (\%) } \\
N=112\end{array}$ & p-value \\
\hline \multicolumn{4}{|l|}{ Patients } \\
\hline \multicolumn{4}{|l|}{ Age: } \\
\hline$-<70$ years & $22(62.9)$ & $71(63.4)$ & 1.0000 \\
\hline$-\geq 70$ years & $13(37.1)$ & $41(36.6)$ & \\
\hline \multicolumn{4}{|l|}{ Gender: } \\
\hline - Male & $24(68.6)$ & $62(55.4)$ & 0.1764 \\
\hline - Female & $11(31.4)$ & $50(44.6)$ & \\
\hline \multicolumn{4}{|l|}{ Smoking status: } \\
\hline - Current/former smoker & $30(85.7)$ & $89(79.5)$ & 0.4709 \\
\hline - Never smoker & $5(14.3)$ & $23(20.5)$ & \\
\hline \multicolumn{4}{|l|}{ ECOG PS: } \\
\hline-0 & $21(60.0)$ & $48(42.9)$ & 0.0841 \\
\hline$-\geq 1$ & $14(40.0)$ & $64(57.1)$ & \\
\hline \multicolumn{4}{|l|}{ Antibiotics: } \\
\hline -Yes & $5(14.3)$ & $14(12.5)$ & 0.7768 \\
\hline- No & $30(85.7)$ & $98(87.5)$ & \\
\hline \multicolumn{4}{|l|}{ Steroids: } \\
\hline - Yes & $0(0.0)$ & $23(20.5)$ & $0.0023^{\dagger}$ \\
\hline- No & $35(100.0)$ & 89 (79.5) & \\
\hline \multicolumn{4}{|l|}{ Acetilsalicilic acid: } \\
\hline - Yes & $12(34.3)$ & $25(22.3)$ & 0.1822 \\
\hline- No & $23(65.7)$ & $87(76.7)$ & \\
\hline \multicolumn{4}{|l|}{ Metformin: } \\
\hline -Yes & $3(8.5)$ & $10(8.9)$ & 1.0000 \\
\hline- No & $32(91.4)$ & $102(91.1)$ & \\
\hline \multicolumn{4}{|l|}{ Statins: } \\
\hline - Yes & $6(17.1)$ & $15(13.4)$ & 0.5858 \\
\hline- No & $29(82.9)$ & 97 (86.6) & \\
\hline \multicolumn{4}{|l|}{ Tumor } \\
\hline \multicolumn{4}{|l|}{ Histology: } \\
\hline - Squamous NSCLC & $8(22.9)$ & $31(27.7)$ & 0.6645 \\
\hline - Nonsquamous NSCLC & $27(77.1)$ & $81(72.3)$ & \\
\hline \multicolumn{4}{|l|}{ Stage at diagnosis: } \\
\hline - Nonmetastatic & $12(34.3)$ & $48(42.9)$ & 0.4332 \\
\hline - Metastatic & $23(65.7)$ & $64(57.1)$ & \\
\hline \multicolumn{4}{|l|}{ PD-L1 expression: } \\
\hline - Positive & $11(31.4)$ & $25(22.3)$ & 0.1780 \\
\hline - Negative & $7(20.0)$ & $37(33.1)$ & \\
\hline - Unknown & $17(48.6)$ & $50(44.6)$ & \\
\hline \multicolumn{4}{|l|}{$\mathrm{N}$ of metastatic sites: } \\
\hline-1 & $3(8.6)$ & $14(12.5)$ & 0.7631 \\
\hline$-\geq 2$ & $32(91.4)$ & $98(87.5)$ & \\
\hline \multicolumn{4}{|l|}{ Liver metastases: } \\
\hline - Present & $6(17.1)$ & $25(22.3)$ & 0.6377 \\
\hline - Absent & $29(82.9)$ & $87(77.7)$ & \\
\hline
\end{tabular}

†Statistically significant p-value.

CR: Complete response; CT: Chemotherapy; ECOG: Eastern Cooperative Oncology Group; ICl: Immune checkpoint inhibitor; LTB: Long-term benefit; N: Number; NLR: Neutrophil/lymphocyte ratio; NSCLC: Non-small-cell lung cancer; P: Progressor; PD: Progressive disease; PR: Partial response; PS: Performance status; SD: Disease stability; STB: Shortterm benefit. 
Table 1. Univariate analyses comparing cases with long-term benefit and controls in the global population (cont.).

\begin{tabular}{|c|c|c|c|}
\hline Characteristics & $\begin{array}{l}\text { LTB, N (\%) } \\
\mathrm{N}=35\end{array}$ & $\begin{array}{l}\mathrm{STB}+\mathrm{P}, \mathrm{N}(\%) \\
\mathrm{N}=112\end{array}$ & p-value \\
\hline$-<5$ & $29(82.9)$ & $71(63.4)$ & $0.0378^{\dagger}$ \\
\hline \multicolumn{4}{|l|}{ Treatment } \\
\hline \multicolumn{4}{|l|}{ Response to previous $\mathrm{CT}$ : } \\
\hline \multicolumn{4}{|l|}{ Response to subsequent CT: } \\
\hline - Yes & 0 & 8 & 0.5601 \\
\hline- No & 3 & 22 & \\
\hline \multicolumn{4}{|l|}{$\mathrm{ICl}$ agent: } \\
\hline - Anti-PD-1 & $23(65.7)$ & $64(57.1)$ & 0.4332 \\
\hline \multicolumn{4}{|l|}{ ICI line: } \\
\hline - First & $7(20.0)$ & $12(10.7)$ & 0.1592 \\
\hline$-\geq$ Second & $28(80.0)$ & $100(89.3)$ & \\
\hline \multicolumn{4}{|l|}{ Grade $\geq 2$ toxicity: } \\
\hline -Yes & $24(68.6)$ & $75(67.0)$ & 1.0000 \\
\hline- No & $11(31.4)$ & $37(33.0)$ & \\
\hline \multicolumn{4}{|l|}{ Radiotherapy: } \\
\hline -Yes & $7(20.0)$ & $22(19.6)$ & 1.0000 \\
\hline- No & $28(80.0)$ & $90(80.4)$ & \\
\hline \multicolumn{4}{|l|}{ First response: } \\
\hline
\end{tabular}

†Statistically significant p-value.

CR: Complete response; CT: Chemotherapy; ECOG: Eastern Cooperative Oncology Group; ICl: Immune checkpoint inhibitor; LTB: Long-term benefit; N: Number; NLR: Neutrophil/lymphocyte ratio; NSCLC: Non-small-cell lung cancer; P: Progressor; PD: Progressive disease; PR: Partial response; PS: Performance status; SD: Disease stability; STB: Shortterm benefit.

\section{Table 2. Multivariate analysis comparing cases with long-term benefit and controls ${ }^{\dagger}$ in the global population.}

\begin{tabular}{|c|c|c|}
\hline Variable & OR for LTB $(95 \% \mathrm{Cl})$ & p-value \\
\hline NLR $(\geq 5$ vs $<5)$ & $0.630(0.204-1.949)$ & 0.4225 \\
\hline Steroids (no vs yes) & $3.673(0.725-18.621)$ & 0.1162 \\
\hline ECOG PS (0 vs $\geq 1)$ & $1.007(0.256-4.539)$ & 0.9877 \\
\hline First response to ICI (SD/PD vs CR/PR) & $1.077(0.258-4.507)$ & 0.9191 \\
\hline Best response to ICI (SD/PD vs CR/PR) & $0.124(0.034-0.453)$ & $0.0016^{\ddagger}$ \\
\hline \multicolumn{3}{|c|}{$\begin{array}{l}\text { †Short-term benefit + progressor. } \\
\text { ¥Statistically significant p-value. } \\
\text { CR: Complete response; ECOG: Eastern Cooperative Oncology Group; ICI: Immune checkpoint inhibitor; LTB: Long-term benefit; NLR: Neutrophil/lymphocyte ratio; OR: Odds ratio; P: } \\
\text { Progressor; PD: Progressive disease; PR: Partial response; PS: Performance status; SD: Disease stability; STB: Short-term benefit. }\end{array}$} \\
\hline
\end{tabular}

\section{Comparison between cases with LTB \& STB}

The subpopulation of patients obtaining a benefit from IO (CR or PR or SD) was composed by 80 subjects. As documented in general population, patients with LTB from IO showed a significantly longer median PFS (21.0 

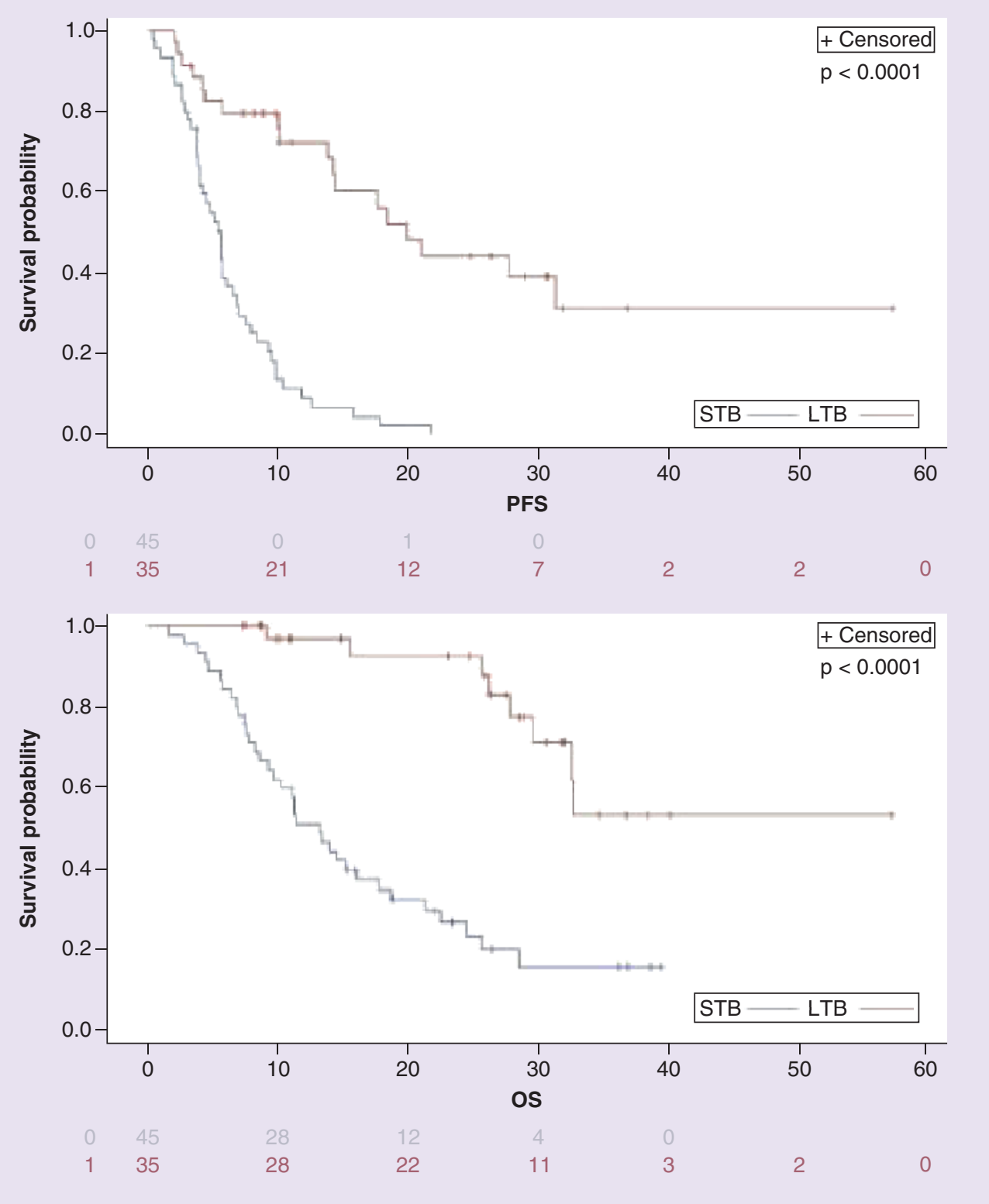

Figure 2. Kaplan-Meier curves of progression-free and overall survival in cases with long- and short-term benefits. LTB: Long-term benefit; OS: Overall survival; PFS: Progression-free survival; STB: Short-term benefit.

vs 5.3 months, $\mathrm{p}<0.0001$ ) and OS (not reached vs 13.2 months, $\mathrm{p}<0.0001$ ) than STB cases, as reported in Figure 2.

Median PFS of either previous or subsequent line of therapy was similar between cases with LTB and STB ( $\mathrm{p}=0.0593$ and 0.5000 , respectively).

The only variable showing a different distribution between LTB and STB patients was the evidence of CR or PR as best response (OR for CR or PR vs SD: 2.629; 95\% CI: 1.051-6.579; $\mathrm{p}=0.0427$ ). This result retained significance after correction for the effect of steroids at multivariate analysis (OR for SD or PD vs CR or PR: 0.356 ; 95\% CI: 0.140-0.906; $\mathrm{p}=0.0303)$. Detailed results are reported in Tables $3 \& 4$. 
Research Article Galli, Proto, Signorelli et al.

Table 3. Univariate analyses comparing cases with long- and short-term benefits.

\begin{tabular}{|c|c|c|c|}
\hline Characteristics & $\begin{array}{l}\text { LTB, N (\%) } \\
\mathrm{N}=35\end{array}$ & $\begin{array}{l}\text { STB, } N(\%) \\
\mathrm{N}=45\end{array}$ & $\mathrm{p}$-value \\
\hline \multicolumn{4}{|l|}{ Age: } \\
\hline$-\geq 70$ years & $13(37.1)$ & $20(44.4)$ & \\
\hline \multicolumn{4}{|l|}{ Gender: } \\
\hline \multicolumn{4}{|l|}{ Smoking status: } \\
\hline - Current/former smoker & $30(85.7)$ & $37(82.2)$ & 0.7663 \\
\hline - Never smoker & $5(14.3)$ & $8(17.8)$ & \\
\hline \multicolumn{4}{|l|}{ ECOG PS: } \\
\hline-0 & $21(60.0)$ & $29(64.4)$ & 1.0000 \\
\hline- No & $30(85.7)$ & $41(91.1)$ & \\
\hline \multicolumn{4}{|l|}{ Steroids: } \\
\hline - Yes & $0(0.0)$ & $5(11.1)$ & 0.0643 \\
\hline- No & $35(100.0)$ & $40(88.9)$ & \\
\hline \multicolumn{4}{|l|}{ Acetilsalicilic acid: } \\
\hline - Yes & $12(34.3)$ & $12(26.7)$ & 0.4733 \\
\hline- No & $23(65.7)$ & $33(73.3)$ & \\
\hline \multicolumn{4}{|l|}{ Metformin: } \\
\hline - Yes & $3(8.5)$ & $7(15.6)$ & 0.5001 \\
\hline- No & $32(91.4)$ & $38(84.4)$ & \\
\hline - Nonsquamous NSCLC & $27(77.1)$ & $30(66.7)$ & \\
\hline \multicolumn{4}{|l|}{ Stage at diagnosis: } \\
\hline - Nonmetastatic & $12(34.3)$ & $21(46.7)$ & 0.3603 \\
\hline - Metastatic & $23(65.7)$ & $24(53.3)$ & \\
\hline \multicolumn{4}{|l|}{ PD-L1 expression: } \\
\hline - High & $11(31.4)$ & $13(28.9)$ & 0.3781 \\
\hline - Low/negative & $7(20.0)$ & $15(33.3)$ & \\
\hline - Unknown & $17(48.6)$ & $17(37.8)$ & \\
\hline \multicolumn{4}{|l|}{$\mathrm{N}$ of metastatic sites: } \\
\hline-1 & $3(8.6)$ & $7(15.6)$ & 0.5001 \\
\hline$-\geq 2$ & $32(91.4)$ & $38(84.4)$ & \\
\hline \multicolumn{4}{|l|}{ Liver metastases: } \\
\hline - Present & $6(17.1)$ & $6(13.3)$ & 0.7555 \\
\hline - Absent & $29(82.9)$ & 39 (86.7) & \\
\hline \multicolumn{4}{|l|}{ NLR: } \\
\hline$-<5$ & 29 (82.9) & $35(77.8)$ & 0.7790 \\
\hline$-\geq 5$ & $6(17.1)$ & $10(22.2)$ & \\
\hline
\end{tabular}




\begin{tabular}{|c|c|c|c|}
\hline Characteristics & $\begin{array}{l}\text { LTB, N (\%) } \\
\mathrm{N}=35\end{array}$ & $\begin{array}{l}\text { STB, N (\%) } \\
\mathrm{N}=45\end{array}$ & $\mathrm{p}$-value \\
\hline \multicolumn{4}{|l|}{ Treatment } \\
\hline \multicolumn{4}{|l|}{ Response to previous $\mathrm{CT}$ : } \\
\hline - Yes & 6 & 15 & 0.1176 \\
\hline - No & 22 & 22 & \\
\hline - No & 3 & 13 & \\
\hline \multicolumn{4}{|l|}{$\mathrm{ICl}$ agent: } \\
\hline - Anti-PD-1 & $23(65.7)$ & $22(48.9)$ & 0.1741 \\
\hline - Anti-PD-L1 & $11(31.4)$ & $21(46.7)$ & \\
\hline - Anti-PD-L1/PD-1+CTLA4 & $0(0.0)$ & $2(4.4)$ & \\
\hline$-\geq$ Second & $28(80.0)$ & $39(86.7)$ & \\
\hline \multicolumn{4}{|l|}{ Grade $\geq 2$ toxicity: } \\
\hline - Yes & $24(68.6)$ & $16(35.6)$ & 0.8128 \\
\hline - No & $11(31.4)$ & $29(64.4)$ & \\
\hline \multicolumn{4}{|l|}{ Radiotherapy: } \\
\hline - Yes & $7(20.0)$ & $13(28.9)$ & 0.4401 \\
\hline - No & $28(80.0)$ & $32(71.1)$ & \\
\hline \multicolumn{4}{|l|}{ First response: } \\
\hline$-C R / P R$ & $12(34.3)$ & $10(22.2)$ & 0.3135 \\
\hline$-S D / P D$ & $23(65.7)$ & $35(77.8)$ & \\
\hline
\end{tabular}

† Statistically significant $\mathrm{p}$-value.

CR: Complete response; CT: Chemotherapy; ECOG: Eastern Cooperative Oncology Group; ICl: Immune checkpoint inhibitor; LTB: Long-term benefit; N: Number; NLR: Neutrophil/lymphocyte ratio; NSCLC: Non-small-cell lung cancer; PD: Progressive disease; PR: Partial response; PS: Performance status; SD: Disease stability; STB: Short-term benefit.

Table 4. Multivariate analysis comparing cases with long- and short-term benefit. Variable

Steroids (no vs yes)

Best response to ICI (SD vs CR/PR)
OR for LTB $(95 \% \mathrm{Cl})$

$2.595(0.444-15.154)$

$0.356(0.140-0.906)$ p-value

0.2895

0.0303

CR: Complete response; ICI: Immune checkpoint inhibitor; LTB: Long-term benefit; OR: Odds ratio; PD: Progressive disease; PR: Partial response; SD: Disease stability.

\section{Comparison between LTB \& P}

The subgroup of cases obtaining PD as best response to IO, thus defined as P, were 67. As expected, the difference in survival became even more evident comparing patients with LTB and P (median PFS: 21.0 vs 1.5 months, $\mathrm{p}<0.0001$; median OS: not reached vs 5.0 months, $\mathrm{p}<0.0001$; survival curves not shown in Figures).

Similarly to previous comparisons, no difference in PFS was documented considering either previous or subsequent line of treatment $(\mathrm{p}=0.3241$ and 0.5452 , respectively).

A differential distribution between LTB and P subgroups was confirmed for NLR ( $p=0.0046)$, for the use of steroids ( $p=0.0162)$, and for the evidence of CR or PR as first $(p<0.0001)$ and best response to IO $(p<0.0001)$. In addition to previously reported findings, also PS at the beginning of IO was significantly different between the two categories of patients (OR: 3.789; 95\% CI: 1.603-8.957; $\mathrm{p}=0.0027$; data not shown in Tables).

In this case, multivariate analysis could not be performed due to the limited number of patients in each cohort and to the quasi-complete separation in the distribution of analyzed categories between the subpopulations. 


\section{Discussion}

The superiority of ICIs over previous standard of care for NSCLC has been evidenced in various disease settings and the indications for IO are consequently broadening. IO is generally well tolerated, but serious and even fatal adverse events, although uncommon, have been described [15,16]. A comprehensive characterization of the subgroup of NSCLC patients with exceptional advantage from treatment is lacking, differently from other malignancies like metastatic melanoma [17]. This could be helpful in personalizing the therapeutic approach particularly in difficult cases, with potential contraindications or risk factors of toxicities from IO. On the other hand the event of primary resistance to IO, up to its worst expression arising as hyperprogression, is well known [18,19]. In such a perspective, simple clinical variables would be more useful in everyday practice than complex and expensive molecular profiles, to identify the patients as best candidates to gain benefit from IO.

In this work, we analyze a wide range of demographic, clinical and pathological variables of metastatic NSCLC patients, in order to investigate their association with LTB from IO.

The empirical definition set for LTB is based on PFS data by trials with IO in different lines (e.g., median PFS of pembrolizumab as first line 10.3 months, as second or more advanced line 3.9 months; median PFS of nivolumab as second or more advanced line ranging from 2.3 months in squamous NSCLC to 3.5 months in nonsquamous NSCLC) [2-5].

As our patients were mostly pretreated when they received IO, the cut-off of 12 months for PFS is three- to six-times longer than the median one reported in regulatory trials, thus fitting to define an effective LTB.

In our case series, LTB is confirmed to be uncommon, being registered in 35 out of 147 cases. This observation is in line with clinical practice and literature data in both NSCLC and other malignancies like metastatic melanoma, where the phenomenon of LTB has been widely studied. Another point in common with melanoma is the improvement in objective response to ICIs during time, with the possibility of conversion either from PD to SD or PR, or from SD to PR, or from PR to CR. This phenomenon is peculiar of IO and has been described in many trials, most commonly with anti-CTLA4 agents $[19,20]$.

Despite these similarities, several differences between NSCLC and melanoma emerge from this work. First of all, prolonged SD is a common pattern of response to ICIs in metastatic melanoma, and patients with long-term SD are a significant proportion of those obtaining LTB [21]. In the present case series of NSCLC patients, LTB seems to be more associated to objective response (CR or PR), while the phenomenon of sustained RECIST stability is relatively less common. Furthermore, disease shrinkage at radiologic evaluation can be evidenced at a quite early point from the beginning of treatment, as median time to best response observed in our case series is significantly shorter (1.8 months) than that described for different diseases [21-23]. Nonetheless, the possibility of a delayed response to IO is confirmed, as best response was registered up to 17.5 months from the beginning of treatment in patients with LTB.

A second point to be underlined pertains to pseudoprogression. This phenomenon has been well described in other malignancies, where lesions sometimes show an initial dimensional or numeric increase in the first phase of IO and subsequently undergo a response which can be prolonged [24-27]. According to this definition, some pseudoprogressions have been evidenced also in our patients, as 14 cases with PD at first evaluation showed at least an SD as best response. Nonetheless, only two cases with LTB had PD as first response; in both these patients, best response was PR. Such an observation supports the possibility of pseudoprogression in NSCLC patients, but this phenomenon seems to be rarely associated to LTB, unless it rapidly converts to an impressive response at subsequent evaluations. In other words, patients with upfront PD to IO may not be excluded from a potential subsequent response, but the benefit they gain from ICIs appears hardly ever substantial. This supports the current tendency to administer IO beyond a first PD, in case of absence of toxicity and evidence of clinical benefit, but may also help clinicians to predict tumor behavior and to plan in advance subsequent therapeutic approaches.

In the global population also first response appears associated to LTB, in univariate but not in multivariate analyses. This observation can be interpreted in relation to best response, whose strong association to LTB likely shades the effects of other variables. First response itself can help in identifying the best candidates to obtain LTB from IO at a very early time point, but the existence of a limited number of discordances between first and best responses (included pseudo-Ps and cases with response improvement in time) prevents from drawing definitive evaluations from first response.

PD-L1 expression is not associated to LTB in our case series. This is in line with recent data, showing a potential benefit from IO also in patients with low/absent PD-L1 expression [28]. However, data about PD-L1 are 
weakened by a significant amount of missing cases and different methods of evaluation. Tumor mutational burden is not available for our cases, as it is not routinely assessed in clinical practice, so its potential association to the phenomenon of LTB remains to be investigated.

Among clinical variables, NLR and steroid uses at the beginning of IO show an association to LTB in univariate analysis, although their significance is lost after correction for the effects of objective response in multivariate analyses. Nonetheless, it has to be underlined that none of the patients with LTB received steroids during the first month after IO administration. This is in line with recent data, showing that early steroid prescription can impair the efficacy of IO [29-32]. On the other hand, differently from literature data, antibiotic use was equally distributed between the patients with or without LTB, not supporting the hypothesis of a detrimental effect at least on the phenomenon exceptional response. It is worth to mention that also other recent works suggest that antibiotics may have a prognostic rather than a predictive impact in NSCLC patients, identifying the subgroup entailing the worst prognosis due to infections and comorbidities [33-37]. In regards to the topic of radiotherapy, the potential synergy between radiation and IO, recently hypothesized with the theoretical basis of the abscopal effect, has not been evidenced in this case series, as the distribution of radiotherapy administration was not different after stratification for LTB $[38,39]$. It has to be underlined that heterogeneity in timing of administration, schedules, fractioning, cumulative doses and targets of radiation therapy may have impaired this result. The impact of acetilsalicilic acid, metformin and statins on IO efficacy in NSCLC is still object of research. Regarding aspirin, recent data suggest that platelet could have a protumoral effect through different mechanisms including immunesuppression [40]. This hypothesis is supported by the evidence about the negative impact of high platelet/lymphocyte ratio on the outcome of NSCLC patients treated with ICIs [41]. Therefore, it has been hypothesized that acetilsalicilic acid could revert this process and restore the antitumoral efficacy of IO [42,43]. Statins have been linked to a reduced risk of developing various types of cancer and with a longer survival after the diagnosis of metastatic disease [44]. In the specific field of NSCLC, data are more convincing about the activity in association to anti-EGFR tyrosine kinase inhibitors, in particular for KRAS-mutated tumors [45]. Furthermore, recent preclinical data suggest that statins may boost anticancer immunity through both receptorial and metabolic pathways [46,47]. As regards to metformin, large observational studies have proved its preventive role on various types of cancer [48,49]. Preclinical data have supported that metformin could also enhance the activity of IO through degradation of PD-L1, increase of $\mathrm{CD}^{+}$tumor-infiltrating lymphocytes and reduction of microenvironment hypoxia [50-52]. None of these three pharmacologic agents showed association with LTB from IO in our case series, leaving room to further clinical research on larger populations on such topics.

After analyzing data about the global population, we specifically focused on patients obtaining a benefit with IO $(\mathrm{LTB}+\mathrm{STB})$, excluding patients with PD as best response $(\mathrm{P})$. This analysis had the aim to deepen the factors specifically associated to LTB and discriminate them from those simply related to any kind of benefit to IO. The results of such analysis substantially confirm those obtained in the global population, as the evidence of CR or PR as best response was the only variable significantly associated to LTB phenotype.

Neither in the general population nor in the subgroup of patients obtaining a benefit with IO, could any differences be evidenced in terms of response to previous and subsequent lines of chemotherapy. This is in contrast with recent data, supporting that patients responsive to chemotherapy tend to be more sensitive to subsequent IO, and that, on the other hand, the administration of IO enhances responses to subsequent chemotherapy [53-55]. At least the result about treatment following IO may be explained by the low number of cases receiving further treatment after PD to ICIs. Indeed, most of our patients were pretreated when they received IO and were not amenable to active therapies due to general conditions at the time of PD. Nonetheless, at least in our experience, the phenomenon of LTB does not seem to be influenced by response to subsequent lines of therapy, leading to conclude that the survival benefit observed for such patients is to be ascribed exquisitely to IO efficacy.

A recent work by Gauci et al. investigated the topic of LTB from IO in a heterogeneous cohort of patients enrolled in a Phase I trial [10]. The authors tested the association between different clinico-pathological variables and long-term outcome, finding that the prognosis of complete responders was much more favorable than that of all the other patients including partial responders. This has been the first study emphasizing the predictive impact of radiologic response on long-term prognosis during treatment with ICIs. Nonetheless, its conclusion is likely dependent on the heterogeneity of cancer types included in the study (e.g., hematologic malignancies, melanoma, soft tissue sarcoma). In particular, the inclusion of diseases associated to higher rates of CRs to IO as compared with NSCLC has probably led to the observed impact such variable on prognosis. Our work is the very first analysis of this kind focusing specifically on NSCLC. Differently from Gauci et al., we considered together CRs and PRs as 
CRs are so rare in NSCLC that any statistical evaluation would be impaired. Apart from this inevitable divergence, our findings support the strong role of radiologic response in defining the patients with NSCLC best candidate to gain LTB from IO, underlining the value of this variable independently of other commonly considered factors like smoking status and NLR.

The main limitation of this work consists in its retrospective nature, focusing on a heterogeneous population, treated with different ICIs in various lines of therapy within a wide range of time, during which available agents and guidelines deeply changed. Furthermore, it cannot be excluded that the limited number of cases has impaired the significance of results in particular for multivariate analyses.

Nonetheless, this work has the value of analyzing a comprehensive range of clinico-pathologic variables, which are broadly used and widely available in everyday practice.

\section{Conclusion}

Our results suggest that an LTB from ICIs tends to be more likely in patients obtaining an early response, or at least an initial stability rapidly followed by an objective response. Therefore, clinicians should be encouraged to pursue IO as much as possible in such cases, with an attention in sparing potentially interfering agents like chronic high-dose steroids. On the contrary, the inability of evidencing significant disease shrinkage within approximately the first 2 months from the beginning of IO does not exclude the chance that a patient derives some benefit from treatment, but renders LTB less likely. These evaluations are completely independent from all other demographic, clinical and pathological variables commonly evaluated in NSCLC patients. In such a perspective, the observations herein reported deserve further consideration and validation in larger case series.

\section{Summary points}

- The indications for the use of immunotherapy $(\mathrm{IO})$ in the treatment of non-small-cell lung cancer (NSCLC) are broadening.

- A comprehensive characterization of patients obtaining long-term benefit (LTB) from IO is lacking.

- We retrospectively collected data about all consecutive patients with metastatic NSCLC treated at our institution between April 2013 and July 2017 in order to investigate the existence of variables potentially associated to LTB phenotype.

- Patients obtaining at least stability as best response to 10 and maintaining it for $\geq 12$ months were considered as having an LTB.

- Patients were defined to have an short term benefit (STB) if they obtained at least a stability as best response but maintained it for $<12$ months.

- The first analysis on the global population showed that the evidence of complete or partial response as best response was the only variable significantly associated to LTB phenotype.

- The second analysis limited on patients deriving a benefit from IO (LTB + short-term benefit) confirmed the same result.

- No other clinical, demographic and pathologic variables appeared to be independently associated with LTB phenotype at multivariate analyses.

- Objective disease response could have a role in identifying the patients with metastatic NSCLC having the highest chance of obtaining LTB from IO.

\footnotetext{
Authors' contributions

G Galli, C Proto and G Lo Russo's substantial contributions to the conception or design of the work, the acquisition, analysis or interpretation of data for the work; drafting the work or revising it critically for important intellectual content; final approval of the version to be published; agreement to be accountable for all aspects of the work in ensuring that questions related to the accuracy or integrity of any part of the work are appropriately investigated and resolved. MC Garassino, D Signorelli, M Imbimbo, R Ferrara, A Prelaj, A De Toma, M Ganzinelli, N Zilembo and F de Braud contributed in drafting the work or revising it critically for important intellectual content; final approval of the version to be published.
} 
attended advisory boards and/or provided lectures for the following organizations, from whom received honoraria or education grants: Amgen, AstraZeneca, Boehringer Ingelheim, BMS, Eli Lilly, F Hoffmann-La Roche, Ignyta, Merck Sharp and Dohme, Merck Serono, Novartis, and Pfizer. MC Garassino declares personal financial interests with the following organizations: AstraZeneca, MSD International GmbH, BMS, Boehringer Ingelheim Italia S.p.A, Celgene, Eli Lilly, Ignyta, Incyte, Inivata, Medlmmune, Novartis, Pfizer, Roche and Takeda; she also declares institutional financial interests with the following organizations: Eli Lilly, MSD, Pfizer (MISP), AstraZeneca, MSD International GmbH, BMS, Boehringer Ingelheim Italia S.p.A, Celgene, Ignyta, Incyte, Inivata, Medlmmune, Novartis, Pfizer, Roche, Takeda, Tiziana and Foundation Medicine; she has received research funding from the following organizations: AIRC, AIFA, Italian Moh and TRANSCAN. GL Russo declares travel accommodations and honoraria with AstraZeneca, MSD International GmbH, BMS and Eli Lilly. The authors have no other relevant affiliations or financial involvement with any organization or entity with a financial interest in or financial conflict with the subject matter or materials discussed in the manuscript apart from those disclosed.

No writing assistance was utilized in the production of this manuscript.

\section{Ethical conduct of research}

The authors state that they have obtained appropriate institutional review board approval or have followed the principles outlined in the Declaration of Helsinki for all human or animal experimental investigations. In addition, for investigations involving human subjects, informed consent has been obtained from the participants involved.

\section{References}

Papers of special note have been highlighted as: $\bullet$ of interest; $\bullet \bullet$ of considerable interest

1. Siegel RL, Miller KD, Jemal A. Cancer statistics, 2018. CA Cancer J. Clin. 68(1), 7-30 (2018).

2. Brahmer J, Reckamp KL, Baas P et al. Nivolumab versus docetaxel in advanced squamous-cell non-small-cell lung cancer. N. Engl. J. Med. 373(2), 123-135 (2015).

3. Borghaei H, Paz-Ares L, Horn L et al. Nivolumab versus docetaxel in advanced nonsquamous non-small-cell lung cancer. N. Engl. J. Med. 373(17), 1627-1639 (2015).

4. Herbst RS, Baas P, Kim DW et al. Pembrolizumab versus docetaxel for previously treated, PD-L1-positive, advanced non-small-cell lung cancer (KEYNOTE-010): a randomised controlled trial. Lancet 387(10027), 1540-1550 (2016).

5. Reck M, Rodríguez-Abreu D, Robinson AG et al. Pembrolizumab versus chemotherapy for PD-L1-positive non-small-cell lung cancer. N. Engl. J. Med. 375(19), 1823-1833 (2016).

6. Rittmeyer A, Barlesi F, Waterkamp D et al. Atezolizumab versus docetaxel in patients with previously treated non-small-cell lung cancer (OAK): a Phase III, open-label, multicentre randomised controlled trial. Lancet 389(10066), 255-265 (2017).

7. Imbimbo M, Lo Russo G, Blackhall F. Current status of immunotherapy for non-small-cell lung cancer. Tumori 102(4), 337-351 (2016).

8. Proto C, Ferrara R, Signorelli D et al. Choosing wisely first line immunotherapy in non-small cell lung cancer (NSCLC): what to add and what to leave out. Cancer Treat. Rev. 75, 35-51 (2019).

9. McDermott D, Lebbé C, Hodi FS et al. Durable benefit and the potential for long-term survival with immunotherapy in advanced melanoma. Cancer Treat. Rev. 40(9), 1056-1064 (2014).

10. Gauci ML, Lanoy E, Champiat S et al. Long-term survival in patients responding to anti-PD-1/PD-L1 therapy and disease out come upon treatment discontinuation. Clin. Cancer Res. 25(3), 946-956 (2019).

-• Analyzes the link between radiologic response and long-term survival with immunotherapy in a wide range of solid and hematologic malignancies.

11. Califano R, Kerr K, Morgan RD et al. Immune checkpoint blockade: a new era for non-small-cell lung cancer. Curr. Oncol. Rep. 18(9), 59 (2016).

- Summarizes the results of clinical trials with immunotherapy in non-small-cell lung cancer and underlines the need of more efficient tools for patients' selection.

12. Brody R, Zhang Y, Ballas M et al. PD-L1 expression in advanced NSCLC: Insights into risk stratification and treatment selection from a systematic literature review. Lung Cancer 112, 200-215 (2017).

13. Nebot-Bral L, Brandao D, Verlingue L et al. Hypermutated tumours in the era of immunotherapy: the paradigm of personalised medicine. Eur. J. Cancer 84, 290-303 (2017).

14. Bernard-Tessier A, Baldini C, Martin P et al. Outcomes of long-term responders to anti-programmed death 1 and anti-programmed death ligand 1 when being rechallenged with the same anti-programmed death 1 and anti-programmed death ligand 1 at progression. Eur. J. Cancer 101, 160-164 (2018).

- Analyzes the outcome of long-term responders after re-challenge of immunotherapy in different types of solid cancer. 
15. Zhao B, Zhao H, Zhao J. Serious adverse events and fatal adverse events associated with nivolumab treatment in cancer patients: nivolumab-related serious/fatal adverse events. J. Immunother. Cancer 6(1), 101 (2018).

16. Wang DY, Salem JE, Cohen JV et al. Fatal toxic effects associated with immune checkpoint inhibitors: a systematic review and meta-analysis. JAMA Oncol. 4(12), 1721-1728 (2018).

17. Zhao B, Zhao H, Zhao J. Impact of clinicopathological characteristics on survival in patients treated with immune checkpoint inhibitors for metastatic melanoma. Int. J. Cancer 144(1), 169-177 (2019).

18. Lo Russo G, Moro M, Sommariva M et al. Antibody-Fc/FcR interaction on macrophages as a mechanism for hyperprogressive disease in non-small-cell lung cancer subsequent to PD-1/PD-L1 blockade. Clin. Cancer Res. 25(3), 989-999 (2019).

19. Ferrara R, Mezquita L, Texier M et al. Hyperprogressive disease in patients with advanced non-small cell lung cancer treated with PD-1/PD-L1 inhibitors or with single-agent chemotherapy. JAMA Oncol. 4(11), 1543-1552 (2018).

20. Schliep S, Agaimy A, Cavallaro A, Kiesewetter F, Schuler G, Heinzerling L. Concealed complete response in melanoma patients under therapy with immune checkpoint inhibitors: two case reports. J. Immunother. Cancer 6(1), 2 (2018).

21. Hodi FS, O’Day SJ, McDermott DF et al. Improved survival with ipilimumab in patients with metastatic melanoma. N. Engl. J. Med. 363(8), 711-723 (2010).

22. Schachter J, Ribas A, Long GV et al. Pembrolizumab versus ipilimumab for advanced melanoma: final overall survival results of a multicentre, randomised, open-label Phase III study (KEYNOTE-006). Lancet 390(10105), 1853-1862 (2017).

23. Larkin J, Chiarion-Sileni V, Gonzalez R et al. Combined nivolumab and ipilimumab or monotherapy in untreated melanoma. N. Engl. J. Med. 373(1), 23-34 (2015).

24. Reckamp KL. Real-world pseudoprogression: an uncommon phenomenon. J. Thor. Oncol. 13(7), 880-882 (2018).

25. Katz SI, Hammer M, Bagley SJ et al. Radiologic pseudoprogression during anti-PD-1 therapy for advanced non-small-cell lung cancer. J. Thor. Oncol. 13(7), 978-986 (2018).

26. Gandara DR, von Pawel J, Mazieres J et al. Atezolizumab treatment beyond progression in advanced NSCLC: results from the randomized, Phase III OAK study. J. Thorac. Oncol. 13(12), 1906-1918 (2018).

- Reports the results of the OAK trial about the postprogression efficacy and safety of atezolizumab.

27. Kazandjian D, Keegan P, Suzman DL, Pazdur R, Blumenthal GM. Characterization of outcomes in patients with metastatic non-small-cell lung cancer treated with programmed cell death protein 1 inhibitors past RECIST version 1.1-defined disease progression in clinical trials. Semin. Oncol. 44(1), 3-7 (2018).

- Evaluates the outcome of patients with non-small-cell lung cancer treated with immunotherapy beyond progression in clinical trials.

28. Lopes G, Wu Y, Kudaba I et al. Pembrolizumab (pembro) versus platinum-based chemotherapy (chemo) as first-line therapy for advanced/metastatic NSCLC with a PD-L1 tumor proportion score (TPS) $\geq 1 \%$ : open-label, Phase III KEYNOTE-042 study. J. Clin. Oncol. 36(Suppl. 18), LBA4-LBA4 (2018).

29. Remon J, Vilariño N, Reguart N. Immune checkpoint inhibitors in non-small-cell lung cancer (NSCLC): approaches on special subgroups and unresolved burning questions. Can. Treat. Rev. 64, 21-29 (2018).

- Underlines the unresolved topics in immunotherapy for non-small-cell lung cancer, including the characterization of the patients most likely to gain long-term benefit.

30. Martínez Bernal G, Mezquita L, Auclin E et al. Baseline corticosteroids (CS) could be associated with absence of benefit to immune checkpoint inhibitors (ICI) in advanced non-small cell lung cancer (NSCLC) patients. Ann. Oncol. 28, 1323P (2017).

31. Arbour KC, Mezquita L, Long N et al. Deleterious effect of baseline steroids on efficacy of PD-(L)1 blockade in patients with NSCLC. J. Clin. Oncol. 36(28), 2872-2878 (2018).

32. Fucà G, Galli G, Poggi M et al. Modulation of peripheral blood immune cells by early use of steroids and its association with clinical outcomes in patients with metastatic non-small-cell lung cancer treated with immune checkpoint inhibitors. ESMO Open 4(1), e000457 (2019).

33. Routy B, Le Chatelier E, Derosa L et al. Gut microbiome influences efficacy of PD-1-based immunotherapy against epithelial tumors. Science 359(6371), 91-97 (2018).

34. Derosa L, Hellmann MD, Spaziano M et al. Impact of antibiotics on outcome in patients with metastatic renal cell carcinoma treated with immune checkpoint inhibitors. J. Clin. Oncol. 29(6), 1437-1444 (2018).

35. Gopalakrishnan V, Spencer CN, Nezi L et al. Gut microbiome modulates response to anti-PD-1 immunotherapy in melanoma patients. Science 359(6371), 97-103 (2018).

36. Matson V, Fessler J, Bao R et al. The commensal microbiome is associated with anti-PD-1 efficacy in metastatic melanoma patients. Science 359(6371), 104-108 (2018).

37. Galli G, Triulzi T, Proto C et al. Association between antibiotic-immunotherapy exposure ratio and outcome in metastatic non-small-cell lung cancer. Lung Cancer 132, 72-78 (2019). 
38. Mohamad O, Diaz de Leon A, Schroeder S et al. Safety and efficacy of concurrent immune checkpoint inhibitors and hypofractionated body radiotherapy. Oncoimmunology 7(7), e1440168 (2018).

39. Hubbeling HG, Schapira EF, Horick NK et al. Safety of combined PD-1 pathway inhibition and intracranial radiation therapy in non-small-cell lung cancer. J. Thorac. Oncol. 13(4), 550-558 (2018).

40. Rachidi S, Metelli A, Riesenberg B et al. Platelets subvert T cell immunity against cancer via GARP-TGF $\beta$ axis. Sci. Immunol. 2(11), eaai7911 (2017).

41. Diem S, Schmid S, Krapf M et al. Neutrophil-to-lymphocyte ratio (NLR) and platelet-to-lymphocyte ratio (PLR) as prognostic markers in patients with non-small cell lung cancer (NSCLC) treated with nivolumab. Lung Cancer 111, 176-181 (2017).

42. Xu XR, Yousef GM, Ni H. Cancer and platelet crosstalk: opportunities and challenges for aspirin and other antiplatelet agents. Blood 131(16), 1777-1789 (2017).

43. Hamada T, Cao Y, Qian ZR et al. Aspirin use and colorectal cancer survival according to tumor CD274 (programmed cell death 1 ligand 1) expression status. J. Clin. Oncol. 35(16), 1836-1844 (2017).

44. Demierre MF, Higgins PD, Gruber SB et al. Statins and cancer prevention. Nat. Rev. Cancer 5, 930-942 (2015).

45. Fiala O, Pesek M, Finek J et al. Statins augment efficacy of EGFR-TKIs in patients with advanced-stage non-small cell lung cancer harbouring KRAS mutation. Tumor Biol. 36(8), 5801-5805 (2015).

46. Dimitroulakos J, Lorimer IA, Goss G. Strategies to enhance epidermal growth factor inhibition: targeting the mevalonate pathway. Clin. Cancer Res. 12(14), 4427s-4431s (2006).

47. Sarrabayrouse G, Pich C, Teiti I, Tilkin-Mariame AF. Regulatory properties of statins and Rho GTPases prenylation inhibitiors to stimulate melanoma immunogenicity and promote anti-melanoma immune response. Int. J. Cancer 140(4), 747-755 (2016).

48. Evans M, Donnelly LA, Emslie-Smith AM et al. Metformin and reduced risk of cancer in diabetic patients. BMJ 330, 1304-1305 (2005).

49. Pusceddu S, Vernieri C, Di Maio M et al. Metformin use associates with longer progression-free survival of patients with diabetes and pancreatic neuroendocrine tumors receiving everolimus and/or somatostatin analogues. Gastroenterology 155(2), 479-489 (2018).

50. Pereira FV, Campelo L, Melo A et al. Metformin exerts antitumor activity via induction of multiple death pathways in tumor cells and activation of a protective immune response. Oncotarget 9(40), 25808-25825 (2018).

51. Eikawa S, Nishida M, Mizukami S et al. Immune-mediated antitumor effect by Type 2 diabetes drug, metformin. Proc. Natl Acad. Sci. USA 112(6), 1809-1814 (2015).

52. Cha JH, Yang WH, Xia W et al. Metformin promotes antitumor immunity via endoplasmic-reticulum-associated degradation of PD-L1. Mol. Cell 71(4), 606-620.e7 (2018).

53. Kobayashi H, Omori S, Nakashima K et al. Response to the treatment immediately before nivolumab monotherapy may predict clinical response to nivolumab. Int. J. Clin. Oncol. 22(4), 690-697 (2017).

54. Park SE, Lee SH, Ahn JS, Ahn MJ, Park K, Sun JM. Increased response rates to salvage chemotherapy administered after PD-1/PD-L1 inhibitors in patients with non-small cell lung cancer. J. Thorac. Oncol. 13(1), 106-111 (2017).

55. Dwary AD, Master S, Patel A et al. Excellent response to chemotherapy post immunotherapy. Oncotarget 8(53), 91795-91802 (2017). 
